Ann. Sci. forest., 1974, 31 (2), 129-134.

\title{
UN NOUVEL OUTIL POUR L'ÉTUDE DES RACINES ET DE LA RHIZOSPHÈRE : LE MINIRHIZOTRON
}

\author{
A. RIEDACKER \\ avec la collaboration technique de J.-F. Muller \\ Station de Sylviculture et de Production, \\ Centre national de Recherches forestières, I.N.R.A. \\ Champenoux, 54370 Einville
}

\begin{abstract}
RÉSUMÉ
Présentation d'un récipient d'élevage de plants permettant d'observer la croissance, la régénération et la morphogénèse des racines ainsi que leur mycorhization, dans les conditions naturelles ou de laboratoire. Des ablations ou manipulations diverses des racines peuvent être pratiquées au cours du développement de la plante.
\end{abstract}

\section{1. - INTRODUCTION}

Au cours des dernières décennies, le système aérien des végétaux a été beaucoup étudié.

En revanche, les études sur les racines sont plus rares bien que l'on reconnaisse de plus en plus leurs rôles multiples.

Des progrès intéressants ont pu être fait récemment à l'aide des chambres souterraines d'observation des racines, ainsi que le mentionne HoffmanN (1974), dans sa revue bibliographique. Elles permettent l'observation de la croissance et du comportement des racines des végétaux ligneux dans des conditions très peu différentes des conditions naturelles. Mais leur coût est souvent élevé et il est malheureusement difficile de contrôler les conditions ambiantes (température, humidité) aussi bien du système racinaire que de la partie aérienne.

Aussi pour étudier le déterminisme de la croissance des racines, divers auteurs ont-ils préféré élever des plants en tubes de verre ou de plexiglass transparent inclinés à $45^{\circ}$. Les conditions édaphiques peuvent alors être contrôlées. En revanche, il est difficile de voir précisément comment se constitue ou se reconstitue le système racinaire et impossible d'intervenir à divers moments pour pratiquer des ablations de racines. De plus le remplissage des tubes est délicat, et il faut que les plants ne soient pas très grands. 
D'autres auteurs (Champagnat et Coll. - 1971) ont cherché à remédier à cet inconvénient en plaçant les racines des plants dans une enceinte munie d'un vaporisateur de solution nutritive. On peut alors observer le système racinaire et intervenir à tout moment; il faut toutefois remarquer qu'on s'écarte alors sensiblement des conditions naturelles (absence d'obtsacle, de pression du sol, alimentation constante, absence de mycorhization, etc...).

C'est en cherchant à allier les avantages de ces différentes techniques que nous avons été amenés. à concevoir les minirhizotrons que nous décrivons ci-dessous.

\section{2. - DESCRIPTION DES MINIRHIZOTRONS}

\section{1. - Principe.}

Les minirhizotrons (Fig. 1) sont des parallélipipèdes plats en matière plastique ayant une face amovible en plexiglass transparent (Fig. 1 a'). Ils sont inclinés à $30^{\circ}$ ou $45^{\circ}$ par rapport à la verticale;

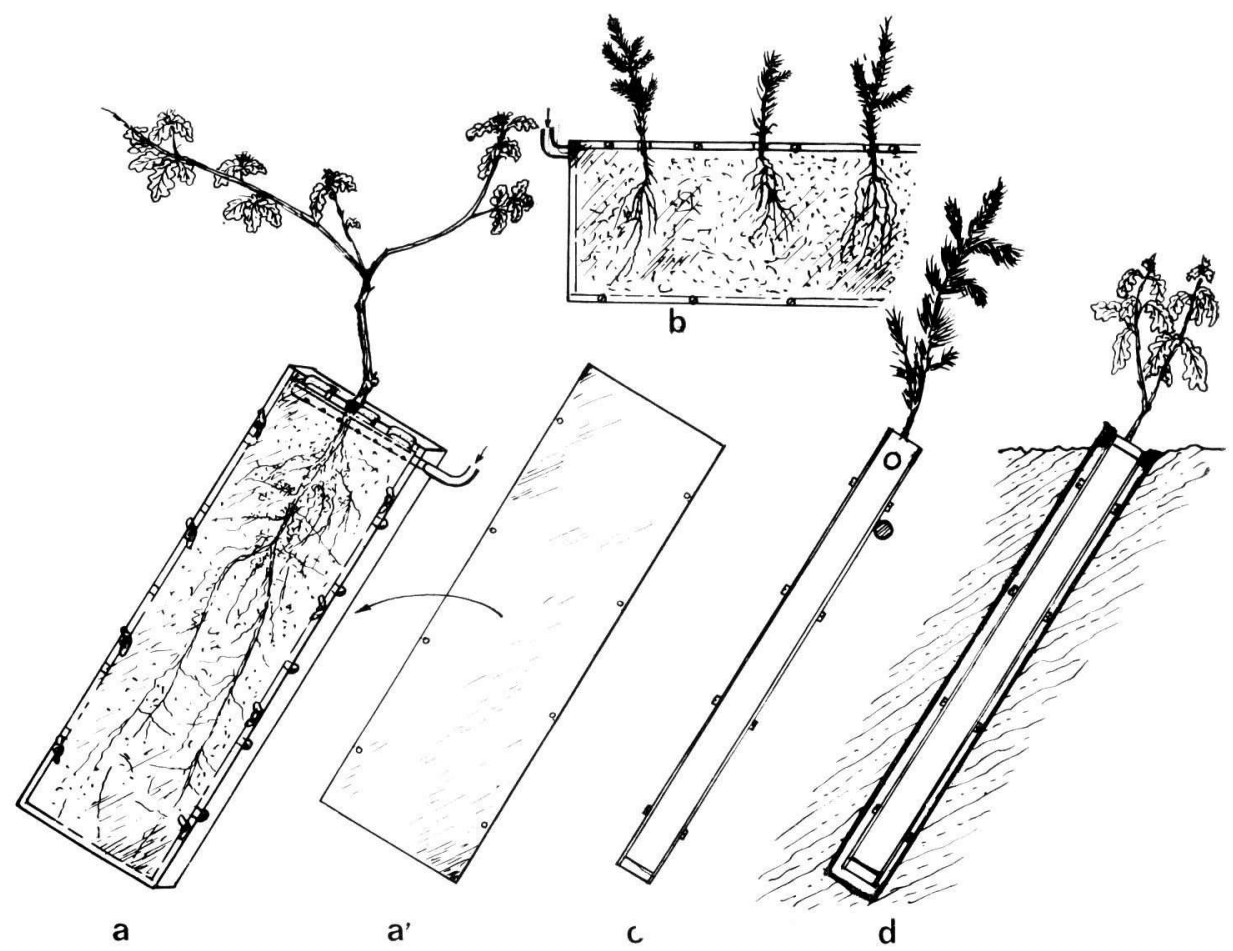

FIG. 1. - Différentes utilisations des minirhizotrons.

$a:$ Jeune plant de chêne dans un minirhizotron « vertical » en matière plastique, avec tube d'alimentation en eau $(\rightarrow)$.

$a^{\prime}:$ Paroi transparente en matière plastique (face inférieure du minirhizotron).

$b$ : Minirhizotron en position horizontale avec plusieurs jeunes plants.

$c$ : Minirhizotron au laboratoire.

$d$ : Un minirhizotron dans son étui placé dans le sol avec joint de caoutchouc pour réduire les fluctuations de température.

\section{Various possibilities of utilization of minirhizotrons.}

$a$ : Single oak seedling in a vertical plastic minirhizotron with a watering tube $(\rightarrow)$

$a$ ' : Transparent plastic cover (lower side of a minirhizotron)

$b$ : Horizontal minirhizotron with several seedlings

$c$ : Lab-minirhizotron

$d$ : A minirhizotron in its case in the soil with a rubber ring do minimize temperature fluctuations. 
sous l'effet du géotropisme, les racines $\left(^{(}\right)$se plaquent contre la face inférieure, àtr avers laquelle il est possible d'observer la croissance et le développement du système racinaire (Fig. 2).

Le fait que cette face soit amovible présente de multiples avantages :

- Les minirhizotrons peuvent être vidés et remplis commodément avec divers substrats (tourbe, argile, etc...).

- Il est possible non seulement d'y faire des semis mais encore d'installer des plants ayant déjà plusieurs années et d'étaler leurs systèmes racinaires contre la plaque de plexiglass sans léser les éventuels « suçoirs » en activité.

- Il est enfin possible d'intervenir au cours du développement des racines pour placer des obstacles sur leur cours, ou de sectionner telle ou telle partie de racine afin de préciser les correlations existant au sein du système racinaire.

La dimension réduite des minirhizotrons permet de les placer soit en pièces climatisées, la partie aérienne et le système racinaire pouvant d'ailleurs croître dans des conditions thermiques différentes et l'alimentation hydrique pouvant être contrôlée, soit en plein air (Fig. 1 d).

Au laboratoire, on peut les mettre dans diverses positions pour voir la réaction des différentes racines à la pesanteur ou pour provoquer très simplement des déformations des extrêmités des racines (crosses, coudes etc...).

\section{2. — Détails techniques}

Nos minirhizotrons ont été réalisés entièrement en matière plastique. Mais on peut aussi envisager de réaliser le cadre en aluminium, matière peu oxydable assurant un bon transfert de chaleur $\left(^{2}\right)$.

\section{Dimensions.}

Elles dépendront de l'utilisation, de la durée des observations et de la taille des plants. Pour nos besoins actuels, nous avons cherché à réaliser des modèles polyvalents de $60 \mathrm{~cm}$ ou $100 \mathrm{~cm}$ de haut, $30 \mathrm{~cm}$ de large et $3 \mathrm{~cm}$ d'épaisseur.

Lorsque les observations sont détaillées et de longue durée, on ne place qu'un seul grand plant par minirhizotrons (Fig. 1 a). Toutefois, si leur système racinaire est très pivotant et peu ramifié, on peut augmenter leur nombre jusqu'à trois.

Si au contraire il s'agit d'observation d'assez courte durée sur des petits plants, notamment lorsqu'il s'agit d'étudier l'aptitude à la régénération des racines, on peut en placer 6 à 10 dans un minirhizotron horizontal (Fig. 1 b).

\section{Système d'alimentation.}

L'alimentation en eau peut être assurée, soit par arrosage manuel à travers les orifices, soit de manière automatique par branchement de la source de solution nutritive sur un tuyau à perforations multiples placé à l'intérieur du minirhizotron (Fig. 1).

\section{Étui des minirhizotrons.}

Pour empêcher la lumière d'arriver aux racines en dehors des périodes d'observation, on peut placer les minirhizotrons dans des sacs en polyethylène noir et éventuellement les enterrer dans le sol. A chaque observation on les déterre. Cette opération devient longue et onéreuse dans le cas d'obser-

(1) Tout au moins celles qui sont sensibles à la pesanteur (qui sont souvent d'ordre 1). Les racines d'ordres plus élevés se trouvent alors de ce fait également près de cette face.

$\left({ }^{2}\right)$ Lorsque ces matières font défaut, on peut utiliser du bois recouvert de résine polyester et du verre. 

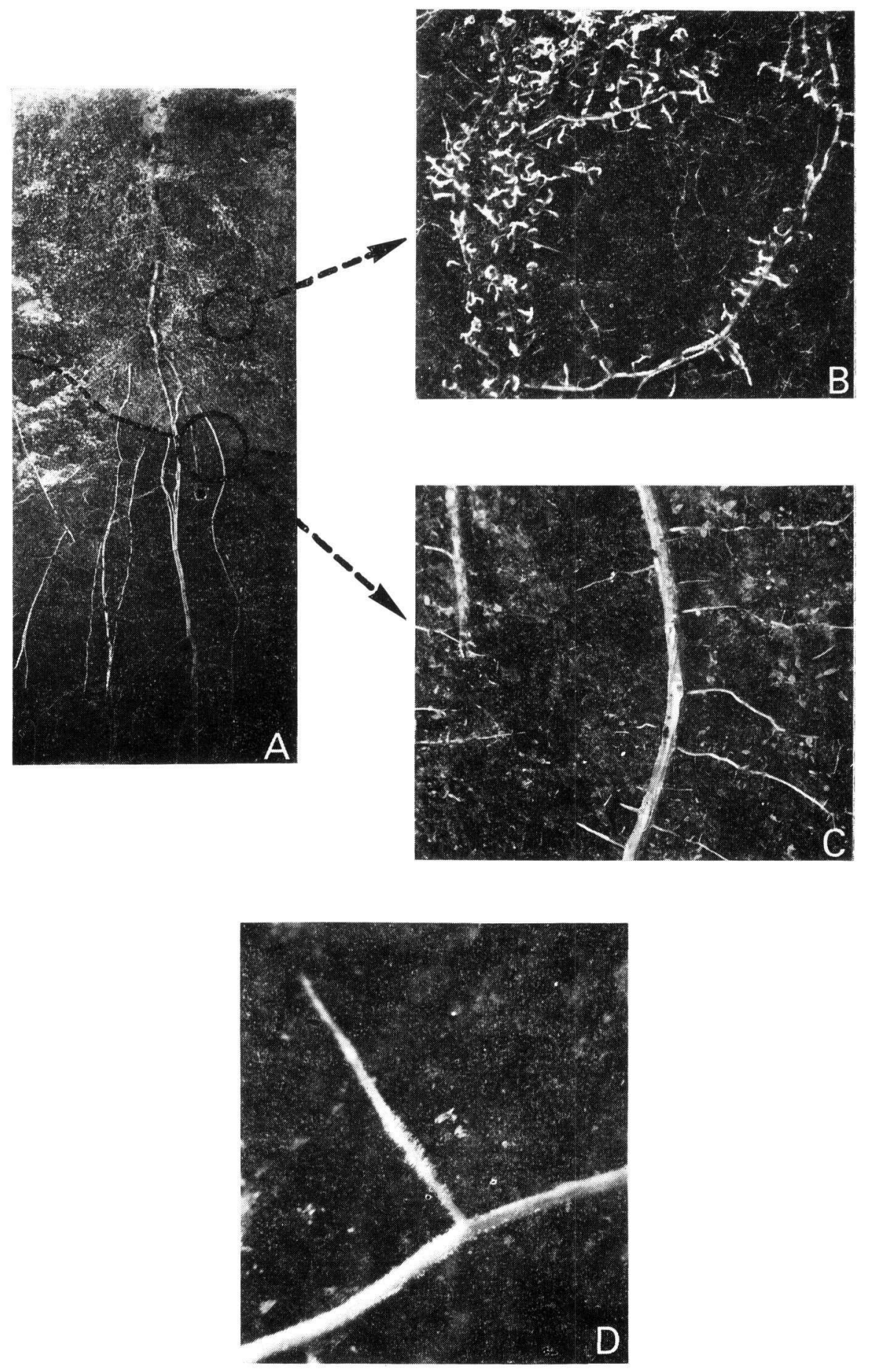

FIG. 2. - Photographie d'un minirhizotron avec un système racinaire de semis de Chêne de 3 ans.

Le pointillé indique la limite de la zone d'extension du mycelium. Celui-ci n'a mycorhizé que les racines de l'année précédente ( 2 b) et non celles de l'année $(2 \mathrm{c})$.

En $(2 \mathrm{~b})$, on peut distinguer à la fois les racines mycorhizées et les filaments myceliens.

(2 d) : Grossissement d'une racine avec ses poils absorbants.

Photograph of a minirhizotron with a root system of a three year old oak seedling.

The dotted line indicates the limits of the mycorrhizae extension.

Roots from previous years with mycorrhizae $(2 \mathrm{~b})$ and currently produced roots without mycorrhizae (2 c).

(2 d) : Root tips with root hairs. 
vations nombreuses et fréquentes. Il est alors préférable d'utiliser des étuis métalliques ou en matière plastique (Fig. $1 \mathrm{~d}$ ) installés à demeure dans le sol et dans lesquels on peut glisser les minirhizotrons. Un joint de caoutchouc isole l'air de l'étui de celui de l'atmosphère extérieure pour éviter les fluctuations de température. Et si l'on désire élever la température du sol, il suffit d'entourer le fourreau d'une résistance chauffante couplée à un thermostat placé dans le minirhizotron, le tout étant isolé du sol.

On peut également y adjoindre une sonde à plâtre et une sonde thermique pour mesurer les variations d'humidité et de température. Lorsque les minirhizotrons sont placés dans des étuis étanches, on peut les plonger dans des bacs d'eau thermostatés. De temps en temps, on vide les étuis pour éliminer l'excès d'eau d'arrosage. Un système de vase communiquant permet d'y maintenir ou d'y faire fluctuer le niveau de l'eau pour simuler les variations d'une nappe d'eau.

\section{Enregistrement des données.}

Soit à l'aide de photographies, (il faut alors supprimer tout éclairage de face pour éviter les reflets, et ne conserver qu'un éclairage latéral rasant), soit par simple recopiage de la croissance à l'aide d'un crayon marqueur sur du rhodoïd.

Le schéma sur rhodoïd est ensuite recopié sur papier calque; il n'est pas possible, en effet, de recopier directement la croissance des racines sur le papier calque, car celui-ci est insuffisamment transparent. Le rhodoïd peut être réutilisé après lavage dans l'alcool.

Les poils absorbants peuvent également être observés : il suffit pour cela de placer les minirhizotrons sous une loupe binoculaire (Fig. 2 d).

\section{3. - UTILISATION ET CRITIQUES}

Les utilisations de ces minirhizotrons sont fort nombreuses ainsi que nous l'avons déjà mentionné. Ils permettent d'étudier la croissance, la morphogénèse, la régénération et la durée de vie des racines de végétaux ligneux pas trop volumineux placés soit dans les conditions naturelles, soit dans des conditions contrôlées.

Dans les conditions naturelles nous avons pu observer (Fig. 2 a et b), l'installation et l'extension des filaments mycorhiziens autour des racines ainsi que la croissance des fines radicelles mycorhizées. Sans doute, les spécialistes des mycorrhizes pourront-ils tirer également parti de cette technique d'observation.

Comme toutes les techniques d'observation des racines, celle-ci comporte également quelques inconvénients. Il existe notamment une discontinuité entre le sol et la plaque de plexiglass. Or, lorsque la pression du sol ou du milieu ambiant diminue, les racines, tout au moins celles des graminées, auraient tendance à s'allonger davantage et à moins croître en diamètre ABdalla et Coll. (1969).

Dans le cas présent, il faut toutefois signaler qu'après l'allongement on a pu constater qu'un certain nombre de racines s'épaississaient.

Lorsque le système racinaire se développe beaucoup, on peut augmenter l'épaisseur du minirhizotron ou mettre une seconde plaque transparente sur la face supérieure. On n'observe alors pas toutes les racines, mais une fraction très importante encore grandement représentative de l'activité de tout le système racinaire (HoffMAnN, 1974).

Il est vrai enfin qu'on force les systèmes racinaires placés en minirhizotron à se développer essentiellement dans un seul plan. Cela nous paraît cependant être un inconvénient extrêmement mineur par rapport aux multiples possibilités d'observation et d'expérimentation que présente par ailleurs l'utilisation des minirhizotrons. 


\section{REMERCIEMENTS}

Nous tenons à remercier l'Association Forêt-Cellulose pour son aide et l'intérêt qu'elle a témoigné pour nos travaux.

\section{SUMMARY}

MINIRHIZOTRONS : A NEW TOOL FOR THE STUDY OF ROOTS AND RHIZOSPHERES.

Minirhizotrons are small containers of various sizes (in the present case $100 \mathrm{~cm} \times 30 \mathrm{~cm} \times 3 \mathrm{~cm}$; Fig. 1) with a plastic transparent side allowing root observation (root growth, regeneration, morphogenesis; Fig. 2). This lower side is detachable, which allows not only easy filling and emptying, but also root manipulation at various stages of roots belonging to seedlings of various ages. Soil and climatic conditions can also be easily controlled.

\section{ZUSAMMENFASSUNG}

\section{EIN NEUES VERFAHREN ZUM STUDIUM DER WURZELN UND DER RHIZOSPHARE :} DER MINIRHIZOTRON.

Das Gefäss (Fig. 1 und 2) erlaubt die Beobachtung des Wachstums, der Regeneration und der Morphogenese der Wurzeln der Gehölze während der ersten Jahre, sowie die Mikorrhysation, sowohl in der Natur, wie im Labor.

Die untere durchsichtige Seite des Minirhizotron (Fig. 1 und a') kann entfernt werden; hierdurch können die Wurzeln gehandhabt oder geschnitten, auch Pflanzen verschiedenen Alters eingesetzt werden; man kann ihn mit Leichtigkeit auffüllen oder entleeren.

\section{RÉFÉRENCES BIBLIOGRAPHIQUES}

Abdalla A.-M. - Hettiaratchi D.R.P. - Reecear 1969. - The mechanics of root growth in granular media. J. agric. Engng. Res. 196914 (3) 236-248.

Champagnat et Coll. 1971. - Communication personnelle.

HoffmanN G. 1974. - Untersuchungs verfahren zur Messung des Wurzelwachstums unter Freiland bedingungen. II : Internationales Symposium Potsdam September 1971. Okologie und Physiologie des Wurzelwachstums; 161-170. 